\title{
VGLL1 expression is associated with a triple-negative basal-like phenotype in breast cancer
}

\author{
María Ángeles Castilla,2, María Ángeles López-García ${ }^{1,2}$, María Reina Atienza', \\ Juan Manuel Rosa-Rosa ${ }^{3}$, Juan Díaz-Martín ${ }^{1,2}$, María Luisa Pecero', Begoña Vieites ${ }^{1,2}$, \\ Laura Romero-Pérez ${ }^{1,2}$, Javier Benítez ${ }^{4}$, Annarica Calcabrini ${ }^{5}$ and José Palacios ${ }^{2,3}$ \\ 'Instituto de Biomedicina de Sevilla-CSIC-Universidad de Sevilla, Hospital Universitario Virgen del Rocío, \\ Department of Pathology, Avda. Manuel Siurot S/N, 41013 Seville, Spain \\ ${ }^{2}$ Red Temática de Investigación Cooperativa en Cáncer (RTICC), ISCIII, Madrid, Spain \\ ${ }^{3}$ Servicio de Anatomía Patológica, Hospital Universitario Ramón y Cajal and Instituto Ramón y Cajal de Investigación \\ Sanitaria (IRYCIS), Madrid, Spain \\ ${ }^{4}$ Human Genetics Group, Departamento de Biología del Cáncer, Spanish National Cancer Research Centre \\ (CNIO)-CIBERER, Madrid, Spain \\ ${ }^{5}$ Department of Technology and Health, Istituto Superiore di Sanità, Rome, Italy
}

Correspondence should be addressed to J Palacios or M Á Castilla Emails jose.palacios@ salud.madrid.org or macastillamoro@ gmail.com

\begin{abstract}
Vestigial-like 1 (VGLL1) is a poorly characterized gene encoding a transcriptional co-activator structurally homologous to TAZ and YAP that modulates the Hippo pathway in Drosophila. In this study, we examined the expression of VGLL1 and its intronic miRNA, miR-934, in breast cancer. VGLL1 and miR-934 expression miRNA profiling was carried out on frozen samples of grade 3 invasive ductal carcinomas. VGLL1 protein was also examined in 433 sporadic and $B R C A 1$-associated breast carcinomas on tissue microarrays. RNA-seq data from The Cancer Genome Atlas (TCGA) was used to confirm differences in VGLL1 and miR-934 expression in different breast cancer subtypes, and to correlate their expression with that of other genes and miRNAs. Of 28 miRNAs differentially expressed in estrogen receptor (ER)-positive and ER-negative grade 3 breast carcinomas, miR-934 was most strongly upregulated in ER-negative carcinomas, and its expression was correlated with that of VGLL1. Nuclear VGLL1 expression was observed in $13 \%$ of sporadic breast carcinomas, and while VGLL1 was only occasionally found in luminal A $(0.70 \%)$ and B $(5.60 \%)$ carcinomas, it was often expressed in HER2-positive (17\%), triple-negative (TN) breast carcinomas ( $>40 \%)$ and BRCA1-associated TN carcinomas ( $>50 \%$ ). These findings were confirmed in the TCGA dataset, which revealed positive associations with luminal progenitor genes (GABRP, SLC6A14, FOXC1, PROM1, and $B B O X 1$ ) and strong negative correlations with ER-associated genes (ESR1, C6ORF211, GATA3, and FOXA1). Moreover, VGLL1 expression was associated with reduced overall survival. In conclusion, VGLL1 and miR-934 are mainly expressed in sporadic and BRCA1-associated TN basal-like breast carcinomas, and their coordinated expression, at least partially mediated by the direct modulation of ESR1, might be involved in the maintenance of a luminal progenitor phenotype.
\end{abstract}

Key Words

- breast cancer

- Vgll1

- miR-934

- basal-like phenotype

- BRCA1 http://erc.endocrinology-journals.org DOI: 10.1530/ERC-13-0485
(C) 2014 Society for Endocrinology Printed in Great Britain
Published by Bioscientifica Ltd
Endocrine-Related Cancer

(2014) 21, 587-599 


\section{Introduction}

Breast cancer is a heterogeneous disease characterized by marked variation in morphological appearance, molecular features, biological behavior, and therapeutic response. Based on the results of gene expression studies, breast carcinomas can be divided into different molecular subtypes with clinical relevance: luminal (A and B), HER2 +, and basal-like breast cancer (Perou et al. 2000, Sorlie et al. 2001, 2003). Although there is significant morphological and molecular heterogeneity among human basal-like breast cancers, most are high-grade, aneuploid, invasive carcinomas, which do not express estrogen receptor (ER) and/or progesterone receptor (PR), or HER2, and that frequently exhibit TP53 mutations (80\%). Moreover, the loss of $R B 1$ and $B R C A 1$ are characteristic features in basal-like tumors (2012). Although complete concordance is lacking, basal-like breast carcinomas are identified pathologically as triplenegative (TN) tumors (i.e. immunohistochemically negative for ER, PR, and HER2) that express some basal markers, such as basal cytokeratins (CK5/6, CK14, and CK17) and/or EGFR (Badve et al. 2011, Toft \& Cryns 2011). Basal-like carcinomas have a worse prognosis compared with other breast tumor subtypes and cannot be treated using ER- or HER2-targeted therapies (reviewed in Toft \& Cryns (2011)).

During the course of an ongoing study into the pattern of miRNA expression in grade 3 invasive breast cancer, we observed marked overexpression of miR-934 in TN breast tumors. The function of this miRNA is unknown, but it is located in intron 4 of the vestigial-like 1 (VGLL1) gene. VGLL1 is a human protein that is related to the Drosophila transcriptional co-activator Vg. VGLL1, which is located on Xq26.3, was identified and isolated from a fetal heart cDNA library and named Tondu (TDU; Vaudin et al. 1999). The homology between $V g$ and TDU is limited to a short domain of 26 amino acids that is essential for its interaction with TEADs (a family of transcriptional factors related to sd - scalloped - in Drosophila). Because $V g$ and TDU have few similarities, $T D U$ is not considered to be orthologous to $V g$, even though functional studies have shown that TDU can rescue $V g$ function, suggesting that these proteins can fulfill analogous functions (Vaudin et al. 1999). In addition, given that TDU can interact with all TEADs, it seems that TDU is indeed a TEAD co-activator (Vaudin et al. 1999).

Although the role of VGLL1 in both normal development and disease is unknown in mammals, some indication of the function of VGLL1 may be found by analyzing $V g$, a transcription factor required for wing cell specification in flies. Non-wing cells are recruited after repression of the Hippo pathway, resulting in the nuclear accumulation of $Y k i$ (TAZ and $Y A P$ in mammals). $Y k i$ forms a complex with $S d$ (TEADs factors in mammals) that directly activates $V g$, triggering the conversion of nonwing cells into wing cells. Interestingly, as $V g$ accumulates, it can substitute $Y k i$ as a $S d$ co-factor that drives $V g$ gene expression (Zecca \& Struhl 2010). It is not known whether similar relationships govern the development of mammalian tissues.

Structural and functional similarities were recently described between the VGLL1-TEAD complex and the complex formed by TEAD and YAP, demonstrating that VGLL1 acts as a specific TEAD co-activator in a manner similar to that of the oncogene YAP (Pobbati et al. 2012). Through his TEAD-interaction domain, YAP enhances multiple processes known to be important for tumor progression and metastasis, including cell proliferation, transformation, migration, and invasion (Lamar et al. 2012). Similarly, it was demonstrated that VGLL1-TEAD complexes promote anchorage-independent cell proliferation and the upregulation of specific proliferation genes in vitro, suggesting a role of VGLL1 in cancer initiation and/or progression (Pobbati et al. 2012). However, no further in vitro or in vivo studies have specifically been analyzed for the role of VGLL1 in human cancer.

Based on the observed overexpression of miR-934 in $\mathrm{TN}$ breast cancer and the location of this miRNA in intron 4 of VGLL1 gene, we analyzed VGLL1 expression in a relatively large cohort of breast cancer patients. In this study, we describe the correlation between VGLL1 and miR-934 expression in breast cancer, and we demonstrate that VGLL1 nuclear protein is more frequently expressed in both sporadic and hereditary forms of TN basal-like breast carcinomas. Studies of several expression databases confirmed our observations and suggest an oncogenic role of VGLL1 in breast cancer.

\section{Materials and methods}

\section{Human tumors}

For miRNA and mRNA analyses, we obtained freshfrozen samples from 60 grade 3 invasive ductal carcinomas (IDCs): $37 \mathrm{ER}+$ and $23 \mathrm{TN}$ breast carcinomas (additional file 2, Supplementary Table 1, see section on

Published by Bioscientifica Ltd. 
supplementary data given at the end of this article). For immunohistochemical analysis, we used 349 formalinfixed paraffin-embedded (FFPE) invasive human breast carcinomas (Table 1). These tumors had been diagnosed between 2006 and 2011, and inclusion criteria were availability of pathological data and tissue for tissue microarray (TMA) construction. To confirm the immunohistochemistry (IHC) results in this series, we obtained FFPE samples from 70 additional $\mathrm{TN}$ breast carcinomas (additional file 2, Supplementary Table 2) and 14 BRCA1associated hereditary breast carcinomas (additional file 2 , Supplementary Table 3). All samples, except the BRCA1associated carcinomas (Palacios et al. 2005), were obtained from the archives of the Department of Pathology, Hospital Virgen del Rocío, Sevilla (Spain). In addition to tumor samples, we used five non-neoplastic breast tissue samples to analyze the immunohistochemical expression pattern of VGLL1 in normal breast.

This study was performed in accordance with standard ethical procedures dictated by Spanish law (Ley de Investigación Orgánica Biomédica, 14th July 2007) and was approved by the Ethics Committee of the Hospital Virgen del Rocío and the Fundación Pública Andaluza para la Gestión de la Investigación en Salud de Sevilla (FISEVI), Spain. Written informed consent was obtained from all patients and all clinical analyses were conducted in accordance with the principles of the Helsinki Declaration.

\section{Breast cancer cell lines}

Human breast carcinoma cell lines with different phenotypes (MCF7, MCF10, T47D, BT474, SKBR3, MDA-MB-231, and BT459) were obtained from the American Tissue Culture Collection (ATCC, Manassas, VA, USA) and were cultured according to the indicated supplier conditions. The cells were maintained as monolayer cultures at $37^{\circ} \mathrm{C}$ in an atmosphere with 5\% $\mathrm{CO}_{2}$. Total RNA from breast carcinoma cell lines was extracted in triplicate from $90 \%$ confluent cells.

\section{Expression of miRNAs and VGLL1 mRNA}

Total RNA was extracted from human breast carcinoma cell lines and selected areas of frozen tissue containing $>70 \%$ tumor cells using the mirVana miRNA Isolation Kit (Ambion, Austin, TX, USA) after having homogenized the tissue for 2 min in lysis buffer using an Ultra-turrax (T10 Basic, IKA, Staufen, Germany), following the manufacturer's protocol. The quantity and quality of the RNA isolated were measured using a NanoDrop ND-100 spectrophotometer (Thermo Scientific, Waltham, MA, USA).

The expression of 667 miRNAs was measured by quantitative real-time PCR (qRT-PCR) using customized TaqMan Human miRNA assays (Human MicroRNA card set v2.0, Applied Biosystems). To validate miR-934 expression, we used qRT-PCR with a TaqMan probe specific for the mature form of this miRNA (Applied Biosystems TaqMan MicroRNA Assays). Details of qRTPCR have been previously described (Castilla et al. 2011, 2012) and are provided as Supplementary information, see section on supplementary data given at the end of this article (additional file 1).

The significance of relative changes in miRNA and mRNA expression between different breast tumor types was determined using the Wilcoxon's rank-sum test, and the $P$ values were corrected using the Benjamini-Hochberg algorithm $(P<0.05)$. These analyses were performed with the Integromics RealTime StatMiner 4.2 Package (Integromics S.L., Granada, Spain), while SPSS (SPSS 17.0, Inc.) was used to perform the Pearson correlation analyses to determine the correlation between VGLL1 and miR-934 gene expression.

\section{Expression of miR-934 and VGLL1 mRNA in The Cancer Genome Atlas dataset}

To validate our results, we used The Cancer Genome Atlas (TCGA) RNA-seq data from the subset of invasive breast carcinomas, which consists of over 500 breast tumor samples. We downloaded the files containing the per-million read count from the TCGA data portal (https://tcga-data.nci.nih.gov/tcga/findArchives.htm) and grouped tumors into subtypes according to the Pam50 signature (basal-like, HER2-enriched, luminal A, luminal B, and normal-like; Supplementary Table 4, see section on supplementary data given at the end of this article).

As no normal tissue match was sequenced for any of the tumors, we normalized the data to allow miRNA and gene samples to be compared (referred to hereafter as locus/loci). Normalization was based on the background expression of the corresponding sample. By using the median of the expressed loci (count $>0$ ) as the expression threshold for each sample, the fold-change in expression in the context of the tumor was obtained by dividing the value for each locus count by the sample threshold. We calculated the median expression for each locus in every subtype and for the global sample set. As the distribution of fold-change expression values was generally skewed (data not shown), the Kruskal-Wallis test was applied

Published by Bioscientifica Ltd. 
Table 1 Association between VGLL1 protein expression and clinicopathological characteristics of infiltrating breast carcinomas

\begin{tabular}{|c|c|c|c|c|}
\hline & Analyzable & $\begin{array}{l}\text { VGLL1-positive } \\
\quad(n=46)\end{array}$ & $\begin{array}{l}\text { VGLL1-negative } \\
\quad(n=303)\end{array}$ & $P$ value* \\
\hline Age (years) & $58.99 \pm 13.54$ & $55.61 \pm 14$ & $59.51 \pm 13.45$ & \\
\hline \multicolumn{5}{|c|}{ Histologic type $(n=349)$} \\
\hline Ductal carcinoma & 310 & $42(13.54 \%)$ & $268(86.45 \%)$ & \\
\hline Lobular carcinoma & 16 & $2(12.50 \%)$ & $14(87.50 \%)$ & 0.7996 \\
\hline Other & 23 & $2(8.69 \%)$ & $21(91.30 \%)$ & \\
\hline \multicolumn{5}{|c|}{ pT (tumor size) $(n=344)$} \\
\hline pT1 & $149(44.31 \%)$ & $19(12.80 \%)$ & $130(87.20 \%)$ & \\
\hline рT2 & $162(47.09 \%)$ & $22(13.60 \%)$ & $140(86.40 \%)$ & 0.9760 \\
\hline pT3 & $22(6.40 \%)$ & $3(13.60 \%)$ & $19(86.40 \%)$ & \\
\hline pT4 & $11(3.20 \%)$ & $1(9.10 \%)$ & $10(90.90 \%)$ & \\
\hline \multicolumn{5}{|l|}{ Nodal status $(n=347)$} \\
\hline $\mathrm{N}$ negative & $181(52.16 \%)$ & $20(11.00 \%)$ & $161(89.00 \%)$ & 0.2666 \\
\hline $\mathrm{N}$ positive & $166(47.83 \%)$ & $25(15.10 \%)$ & $141(84.90 \%)$ & \\
\hline \multicolumn{5}{|c|}{ Pathological stage $(n=344)$} \\
\hline I & $108(31.40 \%)$ & $12(10.40 \%)$ & $96(88.90 \%)$ & \\
\hline II & $146(42.44 \%)$ & $18(15.10 \%)$ & $128(87.70 \%)$ & 0.8180 \\
\hline III & $82(23.84 \%)$ & $13(15.90 \%)$ & $69(84.10 \%)$ & \\
\hline IV & $8(2.33 \%)$ & $1(12.50 \%)$ & $7(87.50 \%)$ & \\
\hline \multicolumn{5}{|c|}{ Histological grade $(n=348)$} \\
\hline Grade 1 & $44(12.61 \%)$ & $0(0.00 \%)$ & $44(100.00 \%)$ & $<0.0001$ \\
\hline Grade 2 & $119(34.12 \%)$ & $7(5.90 \%)$ & $121(94.10 \%)$ & \\
\hline Grade 3 & $185(53.30 \%)$ & $39(21.11 \%)$ & $146(78.90 \%)$ & \\
\hline \multicolumn{5}{|l|}{$\mathrm{KI}-67(n=349)$} \\
\hline $\mathrm{KI}-67<14 \%$ & $170(48.71 \%)$ & $3(1.80 \%)$ & $167(98.20 \%)$ & $<0.0001$ \\
\hline KI-67 14-50\% & $137(39.26 \%)$ & $24(17.50 \%)$ & $113(82.50 \%)$ & \\
\hline $\mathrm{KI}-67 \geq 50 \%$ & $42(12.03 \%)$ & $19(45.20 \%)$ & $23(54.80 \%)$ & \\
\hline \multicolumn{5}{|c|}{ Immunohistochemical markers } \\
\hline \multicolumn{5}{|c|}{$\operatorname{ER}(n=349)$} \\
\hline Negative & $118(33.80 \%)$ & $36(30.50 \%)$ & $82(69.50 \%)$ & $<0.0001$ \\
\hline Positive & $231(66.18 \%)$ & $10(4.30 \%)$ & $213(95.70 \%)$ & \\
\hline \multicolumn{5}{|l|}{$\operatorname{PR}(n=347)$} \\
\hline Negative & $154(44.30 \%)$ & $39(25.30 \%)$ & $115(74.70 \%)$ & $<0.0001$ \\
\hline Positive & $193(56.70 \%)$ & $7(3.60 \%)$ & $186(96.40 \%)$ & \\
\hline \multicolumn{5}{|l|}{$\operatorname{HER} 2(n=348)$} \\
\hline Negative & $259(74.42 \%)$ & $31(12.00 \%)$ & $228(88.80 \%)$ & 0.2405 \\
\hline Positive & $89(25.57 \%)$ & $15(16.90 \%)$ & $74(83.10 \%)$ & \\
\hline \multicolumn{5}{|l|}{$\operatorname{EGFR}(n=347)$} \\
\hline Negative & $328(94.62 \%)$ & $40(12.20 \%)$ & $288(87.80 \%)$ & 0.0749 \\
\hline Positive & $19(5.48 \%)$ & $5(26.30 \%)$ & $14(73.70 \%)$ & \\
\hline \multicolumn{5}{|l|}{ Basal CKs $(n=348)$} \\
\hline Negative & $311(89.36 \%)$ & $28(9.00 \%)$ & $283(91.00 \%)$ & $<0.0001$ \\
\hline Positive & $37(10.60 \%)$ & $18(48.60 \%)$ & $19(51.40 \%)$ & \\
\hline \multicolumn{5}{|l|}{ C-KIT $(n=312)$} \\
\hline Negative & $298(95.55 \%)$ & $33(11.07 \%)$ & $264(88.93 \%)$ & $<0.0002$ \\
\hline Positive & $14(4.45 \%)$ & $7(50 \%)$ & $7(50 \%)$ & \\
\hline \multicolumn{5}{|c|}{ Molecular phenotype $(n=349)$} \\
\hline Luminal A & $142(40.70 \%)$ & $1(0.70 \%)$ & $141(99.30 \%)$ & \\
\hline Luminal B & $54(15.50 \%)$ & $3(5.60 \%)$ & $51(94.40 \%)$ & $<0.0001$ \\
\hline HER $2+/ R E+$ & $28(8.80 \%)$ & $5(17.90 \%)$ & $23(82.10 \%)$ & \\
\hline HER2 & $58(16.60 \%)$ & $10(17.20 \%)$ & $48(82.80 \%)$ & \\
\hline TN-NOS & $32(9.20 \%)$ & $11(34.40 \%)$ & $21(65.60 \%)$ & \\
\hline TN-BL & $35(10.00 \%)$ & $16(45.70 \%)$ & $19(54.30 \%)$ & \\
\hline
\end{tabular}

ER, estrogen receptor; PR, progesterone receptor; EGFR, epidermal growth factor receptor; basal CKs, basal cytokeratins (CK5/6 and/or CK17); TN-NOS, triple-negative not otherwise specified; TN-BL, triple-negative basal-like. * $P$ values were calculated using the Pearson's $\chi^{2}$ test. Bold, $P$ values are statistically significant.

http://erc.endocrinology-journals.org DOI: 10.1530/ERC-13-0485
(C) 2014 Society for Endocrinology Printed in Great Britain
Published by Bioscientifica Ltd. 
to determine differences between subtypes, followed by Mann-Whitney $U$ and Wilcoxon's tests for subtype pairwise analyses. A Pearson's correlation was used to confirm the correlation between VGLL1 and miR-934 expression, as a linear relationship was expected between the two loci. However, a Spearman's correlation was used when interactions between other miRNAs and genes were analyzed, as a monotonic relationship was expected in these cases. Statistics were performed using in-house perl (http://www.perl.org/) scripts and R (http:// www.r-project.org/).

To determine whether a given miRNA could directly interact with a gene, we used data from the microRNA.org database (http://www.microrna.org/microrna/getDownloads.do) which contains published data and several available predictors (Dweep et al. 2011). We evaluated three available predictors (miRNA.org values) considered to be the best tools for making predictions: conservation, energy, and mirSVR score. To determine whether our observations at the mRNA level had a measurable effect at the protein level, we obtained contrast results when compared with those data from the cBioPortal protein array (Cerami et al. 2012), bearing in mind that these arrays contain a limited number of proteins.

In addition, CNA status was checked (Cerami et al. 2012) on 71 samples overexpressing VGLL1 to confirm or discard the association between increased number of copies of $200 \mathrm{~kb}$ region surrounding VGLL1 in chromosome $\mathrm{X}$ and overexpression of VGLL1 and miR-934.

\section{TMA construction and IHC}

Tissue sections from FFPE samples were stained with hematoxylin and eosin and representative areas were selected by three pathologists (J Palacios, M Á Castilla, and B Vieites) to obtain two $1 \mathrm{~mm}$ diameter tissue cores. Different TMAs were constructed with invasive breast carcinomas. IHC was carried out on TMA sections using the Envision method (Dako, Glostrup, Denmark) using primary antibodies against ER, PR, HER2, Ki-67, CK5/6, CK17, EGFR, CKIT, p63, CD3, CD20, and CD45. Myoepithelial staining of p63 was carried out to verify the diagnosis of ductal carcinoma in situ. CD3, CD20, and CD45 were used to identify intraepithelial and stromal lymphocytes. Details of the clones, suppliers, dilutions and scoring criteria used are provided in additional file 2 (Supplementary Table 5 , see section on supplementary data given at the end of this article). Details for microscope, camera, software, and conditions used to capture IHC images are provided as Supplementary information (additional file 1).
Based on recent recommendations (Goldhirsch et al. 2011), breast carcinomas were classified into five IHCbased subtypes as follows: luminal A (ER+ and/or PR+, HER2 -, Ki-67 index <14\%); luminal B/HER2 - (ER+ and/or PR +, HER2 -, Ki-67 index $\geq 14 \%$ ); luminal B/HER2 + (ER + and/or PR +, HER2 +); HER2 (ER - , PR - , and HER2+); and TN (ER-, PR-, and HER2-). TN breast carcinomas that expressed any basal cytokeratin and/or EGFR were termed TN-BL (basal-like), while all other $\mathrm{TN}$ breast carcinomas were termed TN-NOS (not otherwise specified).

VGLL1 protein expression was assessed by IHC using the Envision method (Dako) and VGLL1 rabbit polyclonal antibody (10124-2-AP, Proteintech, Chicago, IL, USA) at 1:50 dilution, after heat-induced retrieval step. Following provider's recommendation, we used prostate carcinomas as positive control.

Vgll1 IHC data and clinicopathological parameters were analyzed using the Pearson's $\chi^{2}$ cross-tabulation test. The $P$ value for the total effect of a factor was calculated from the (partial) likelihood ratio statistic. Analyses were performed with the JMP 8.0 Software Package (JMP Statistical Discovery Software, SAS Institute, Inc., Cary, NC, USA).

\section{Immunofluorescence}

SKBR3 and MCF7 breast cancer cells were seeded on sterile coverslips, fixed with $4 \%$ paraformaldehyde/PBS, permeabilized with $0.1 \%$ Triton X-100/PBS, and blocked with $10 \%$ normal goat serum/PBS. Coverslips were incubated for $2 \mathrm{~h}$ at $37^{\circ} \mathrm{C}$ with a rabbit polyclonal anti-VGLL1 (Proteintech; 1:50 in PBS-1\% BSA), then with a goat anti-rabbit IgG Alexa-Fluor 488 (1:400 in PBS-1\% BSA) for $1 \mathrm{~h}$ at $37^{\circ} \mathrm{C}$. After washing with PBS, the cells were counterstained with $10 \mu \mathrm{g} / \mathrm{ml}$ DAPI/PBS (10 min at $37^{\circ} \mathrm{C}$ ) and coverslips were mounted on slides with ProLong reagent (Life Technologies). The samples were observed and photographed using a Nikon Eclipse 90i fluorescence microscope equipped with Digital Sight DS-Qi1 camera and Nis-Elements BR imaging software (Nikon, Tokyo, Japan).

\section{Survival analysis}

Survival analysis was performed on a group of 212 invasive breast carcinoma patients for whom clinical data were available. The details concerning to therapy received are provided as Supplementary information (additional file 1).

Published by Bioscientifica Ltd. 
The probabilities of overall survival (OS) and diseasefree survival (DFS) were estimated using the Kaplan-Meier method and survival differences determined by the logrank test. Univariate and multivariate analyses of OS and DFS were performed using the Cox proportional hazard model (SPSS 17.0, SPSS, Inc.). OS was defined as the period between the first diagnostic biopsy and death caused by breast cancer-related complications, and DFS as the period between the first diagnostic biopsy and the first local or distant recurrence/relapse.

\section{Results}

\section{Expression of miR-934 and VGLL1 mRNA in grade 3 IDCs}

We first investigated miRNAs that were differentially expressed between $22 \mathrm{ER}+$ and $13 \mathrm{TN}$ grade 3 fresh frozen IDCs. We examined a total of 667 miRNAs in these samples by quantitative RT-PCR using TaqMan miRNA arrays. Expression analysis revealed 28 miRNAs that were differentially expressed between the two groups (false discovery rate (FDR) <0.05), 14 miRNAs that were upregulated and 14 downregulated in TN breast carcinomas. In all cases, at least a twofold difference in expression was detected between the two groups (Table 2).

Interestingly, we identified six upregulated miRNAs that were localized on chromosome $\mathrm{X}$ in TN IDCs (Table 2), four of them being located in the Xq26-28 band (miR-934, miR-505, miR-505*, and miR-18b). The miR-934 was the most strongly upregulated miRNA in TN IDCs (61.5-fold increase with respect to ER + breast carcinomas). The large majority of TN IDCs analyzed expressed miR-934; however, this miRNA was detected in only two of the 22 grade 3 ER + IDCs, in which it was expressed at very low levels.

To validate the upregulation of miR-934 observed in TN IDCs, we analyzed a larger number of fresh-frozen tumor samples. In total, we analyzed 60 grade 3 IDCs by qRT-PCR using a TaqMan probe specific for miR-934, 37 $\mathrm{ER}+$, and 23 TN IDCs. Consistent with our initial result, we observed a significant increase in the expression of miR-934 in TN as opposed to ER + IDCs ( $P=0.013$; Fig. 1a).

As miR-934 is embedded in intron 4 of the VGLL1 gene, we investigated whether VGLL1 expression also varied between these subtypes of grade 3 IDCs. Analysis of VGLL1 mRNA expression by qRT-PCR revealed a marked increase in gene expression in TN (15.66-fold increase, $P=0.001$ ) vs ER + IDCs (Fig. 1b). Moreover, mRNA VGLL1 expression was directly correlated with miR-934 expression (Pearson's $r=0.611, P<0.001$; Fig. 1c).
Table 2 Dysregulated miRNA expression in triple-negative vs luminal grade 3 breast carcinoma

\begin{tabular}{|c|c|c|c|}
\hline MiRNA & Fold-change & Adj. $P$ value & $\begin{array}{c}\text { Chromosome } \\
\text { localization }\end{array}$ \\
\hline miR-934 & 61.50 & $3.50 \times 10^{-3}$ & $X q 26.3$ \\
\hline miR-505 & 25.89 & $4.00 \times 10^{-2}$ & Xq27.1 \\
\hline miR-135b-3p & 15.70 & $2.56 \times 10^{-2}$ & $1 q 32.1$ \\
\hline miR-135b-5p & 9.38 & $4.35 \times 10^{-2}$ & $1 q 32.1$ \\
\hline miR-9-3p & 7.54 & $1.44 \times 10^{-2}$ & $1 q 22$ \\
\hline miR-9-5p & 7.29 & $4.85 \times 10^{-2}$ & $1 q 22$ \\
\hline $\operatorname{miR}-25^{*}$ & 7.11 & $4.84 \times 10^{-2}$ & $7 q 22.1$ \\
\hline $\operatorname{miR}-545^{*}$ & 5.35 & $2.94 \times 10^{-2}$ & Xa13.1 \\
\hline miR-505* & 5.21 & $2.93 \times 10^{-2}$ & Xq27.1 \\
\hline miR-18b & 3.12 & $4.00 \times 10^{-2}$ & Xq26.2 \\
\hline miR-628-3p & 3.06 & $2.93 \times 10^{-2}$ & $15 q 21.3$ \\
\hline miR-18a & 2.92 & $4.00 \times 10^{-2}$ & $13 q 31.3$ \\
\hline miR-146a & 2.41 & $4.00 \times 10^{-2}$ & $5 q 34$ \\
\hline miR-660 & 2.09 & $4.00 \times 10^{-2}$ & Xq11.23 \\
\hline miR-190b & -13.25 & $3.50 \times 10^{-3}$ & $15 q 22.2$ \\
\hline miR-337-5p & -12.14 & $9.74 \times 10^{-3}$ & $14 q 32.3$ \\
\hline miR-943 & $\begin{array}{r}-9.42 \\
-9.42\end{array}$ & $2.93 \times 10^{-2}$ & $4 q 16.3$ \\
\hline miR-526b* & -7.35 & $3.92 \times 10^{-2}$ & $19 q 13.42$ \\
\hline miR-149 & -5.88 & $1.65 \times 10^{-2}$ & $2 \mathrm{q} 27.3$ \\
\hline $\operatorname{miR}-29 c^{*}$ & -4.52 & $2.93 \times 10^{-2}$ & $1 \mathrm{q} 32.2$ \\
\hline miR-342-3p & $\begin{array}{l}-4.12 \\
-3.15\end{array}$ & $4.35 \times 10^{-2}$ & $14 q 32.2$ \\
\hline let-7b & -2.73 & $4.35 \times 10^{-2}$ & $22 q 13.31$ \\
\hline $\operatorname{miR}-29 c$ & -2.44 & $4.64 \times 10^{-2}$ & $1 q 32.2$ \\
\hline miR-425 & -2.40 & $4.35 \times 10^{-2}$ & $3 p 21.31$ \\
\hline miR-103 & -2.30 & $4.35 \times 10^{-2}$ & $5 q 34$ \\
\hline miR-126* & -2.06 & $1.73 \times 10^{-2}$ & $9 q 34.3$ \\
\hline miR-135a* & -2.05 & $2.97 \times 10^{-2}$ & $3 q 21.1$ \\
\hline let-7f & -2.01 & $4.47 \times 10^{-2}$ & $\mathrm{Xq11.22}$ \\
\hline
\end{tabular}

miRNA*, miRNA expressed at low levels relative to the same miRNA in the opposite arm of a hairpin. The significance of the relative changes in miRNA expression between grade 3 breast tumors was determined using the Wilcoxon's rank-sum test. $P$ values were corrected using the BenjaminiHochberg algorithm $(P<0.05)$.

\section{Expression of miR-934 and VGLL1 in the TCGA dataset}

The change in the expression observed in the RNA-seq data confirmed the differential expression of miR-934 and VGLL1 among breast cancer subtypes (additional file 2, Supplementary Table 6 , see section on supplementary data given at the end of this article). Expression of VGLL1 and miR-934 was over 100 times higher in basal-like carcinomas than in all other subtypes. It should be noted that the expression values for both loci were close to 0 for HER2-enriched and luminal A and luminal B tumors, while they were close to twice the tumor background threshold in basal-like tumors. Pairwise analyses (additional file 2, Supplementary Table 7) revealed that the basal-like subtype differed significantly from HER2enriched, luminal A, and luminal B subtypes. Pairwise results appeared to be biased for the normal-like subtype, probably due to the small number of tumors analyzed, and

Published by Bioscientifica Ltd 

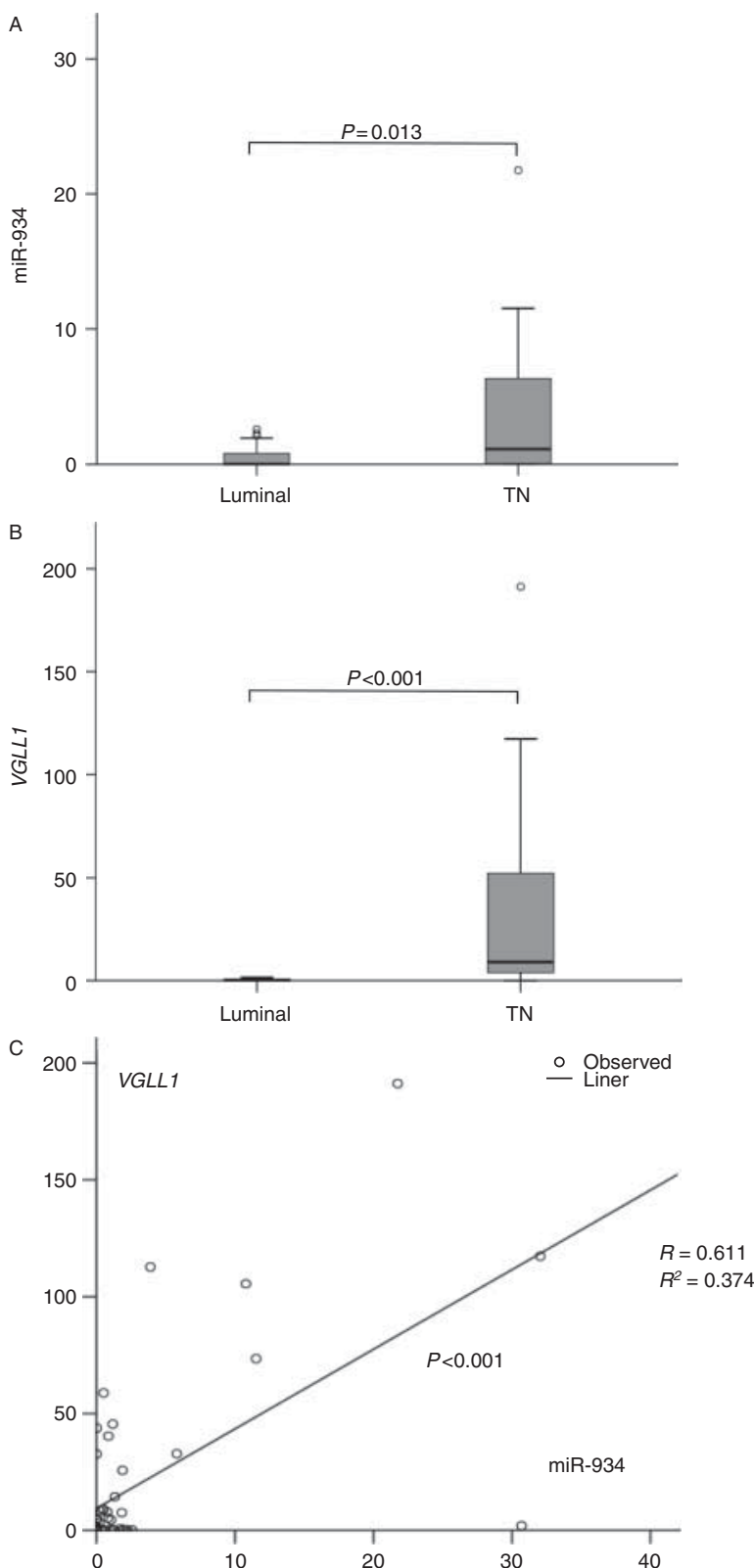

Figure 1

miR-934 and VGLL1 mRNA expression in breast cancer. Expression of miR-934 (upper panel, A) and VGLL1 mRNA (middle panel, B) determined by quantitative RT-PCR in fresh-frozen samples of grade 3 estrogen receptor-positive and triple-negative breast carcinomas. The significance of relative changes was determined by the Wilcoxon's rank-sum test and the $P$ values were corrected using the Benjamini-Hochberg algorithm $(P<0.05)$. The $Y$-axis shows fold changes in relative gene expression. (C) Correlation between miR-934 and VGLL1 expression levels. Pearson correlation coefficients $(R)$ and the level of statistical significance $(P)$ of the correlations were determined.

for the HER2-enriched, luminal A, and luminal B subtypes, probably due to their low levels of expression. There was a significant correlation between VGLL1 and miR-934 expression in this series (Pearson's $r=0.86, P<2.2 \times$ $\left.10^{-16}\right)$, confirming our previous observations. It is important to highlight that none of the 71 samples overexpressing VGLL1 and miR-934 showed alterations in the number of copies of the region surrounding VGLL1.

To determine which genes are candidate targets to be regulated by miR-934 in breast cancer, we assessed the Spearman's correlation between miR-934 and all the genes enclosed in the dataset (not shown). VGLL1 was most strongly correlated with miR-934 (Table 3), supporting the hypothesis of simultaneous transcription. Interestingly, ER (ESR1), FOXA1, and GATA3, which are thought to

Table 3 Top 10 genes most positively and negatively correlated with VGLL1 and miR-934 expression

\begin{tabular}{|c|c|c|}
\hline Gene & $\begin{array}{l}\text { Spearman's } \\
\text { correlation }\end{array}$ & $P$ value \\
\hline \multicolumn{3}{|c|}{ Genes correlated to miR-934 expression } \\
\hline VGLL1 & 0.7824696 & $2.2 \times 10^{-16}$ \\
\hline$G A B R P$ & 0.6591587 & $2.2 \times 10^{-16}$ \\
\hline SFRP1 & 0.6065420 & $2.2 \times 10^{-16}$ \\
\hline PROM1 & 0.5941352 & $2.2 \times 10^{-16}$ \\
\hline ROM1 & 0.5941352 & $2.2 \times 10^{-16}$ \\
\hline$B C L 11 A$ & 0.5871717 & $2.2 \times 10^{-16}$ \\
\hline FOXC1 & 0.5845652 & $2.2 \times 10^{-16}$ \\
\hline$F A B P 7$ & 0.5829631 & $2.2 \times 10^{-16}$ \\
\hline PPP1R14C & 0.5759719 & $2.2 \times 10^{-16}$ \\
\hline$B B O X 1$ & 0.5753999 & $2.2 \times 10^{-16}$ \\
\hline FOXA1 & -0.5591262 & $2.2 \times 10^{-16}$ \\
\hline C6ORF211 & -0.5363153 & $2.2 \times 10^{-16}$ \\
\hline ESR1 & -0.5284415 & $2.2 \times 10^{-16}$ \\
\hline GATA3 & -0.5224655 & $2.2 \times 10^{-16}$ \\
\hline P4HTM & -0.5187309 & $2.2 \times 10^{-16}$ \\
\hline FSIP1 & -0.5091628 & $2.2 \times 10^{-16}$ \\
\hline SIP1 & -0.5091628 & $2.2 \times 10^{-16}$ \\
\hline DNAJC12 & -0.5090977 & $2.2 \times 10^{-16}$ \\
\hline$M L P H$ & -0.5050419 & $2.2 \times 10^{-16}$ \\
\hline TLE3 & -0.4965525 & $2.2 \times 10^{-16}$ \\
\hline \multicolumn{3}{|c|}{ Genes correlated to VGLL1 expression } \\
\hline GABRP & 0.7576318 & $2.2 \times 10^{-16}$ \\
\hline SLC6A14 & 0.7236976 & $2.2 \times 10^{-16}$ \\
\hline FOXC1 & 0.7200038 & $2.2 \times 10^{-16}$ \\
\hline PPP1R14C & 0.7152430 & $2.2 \times 10^{-16}$ \\
\hline SFRP1 & 0.7145003 & $2.2 \times 10^{-16}$ \\
\hline TRIM29 & 0.7101217 & $2.2 \times 10^{-16}$ \\
\hline PROM1 & 0.6993393 & $2.2 \times 10^{-16}$ \\
\hline ROM1 & 0.6993393 & $2.2 \times 10^{-16}$ \\
\hline$F A B P 7$ & 0.6924925 & $2.2 \times 10^{-16}$ \\
\hline$B B O \times 1$ & 0.6924811 & $2.2 \times 10^{-16}$ \\
\hline FOXA1 & -0.627766 & $2.2 \times 10^{-16}$ \\
\hline ESR 1 & -0.625860 & $2.2 \times 10^{-16}$ \\
\hline GATA3 & -0.6243054 & $2.2 \times 10^{-16}$ \\
\hline P4HTM & -0.6065218 & $2.2 \times 10^{-16}$ \\
\hline DNAJC12 & -0.603839 & $2.2 \times 10^{-16}$ \\
\hline$R A B E P 1$ & -0.599712 & $2.2 \times 10^{-16}$ \\
\hline TLE3 & -0.5975988 & $2.2 \times 10^{-16}$ \\
\hline C6ORf211 & -0.5824467 & $2.2 \times 10^{-16}$ \\
\hline SLC16A6 & -0.5818284 & $2.2 \times 10^{-16}$ \\
\hline FSIP1 & -0.5800131 & $2.2 \times 10^{-16}$ \\
\hline
\end{tabular}


determine a luminal lineage (Perou et al. 2000, Sorlie et al. 2001), were among the top ten negatively correlated genes (Table 3). The predictions (microRNAs.org database) indicated that miR-934 may interact directly with the regulatory regions of $E S R 1$, while there were no indications of direct interactions between this miRNA and the regulatory regions of FOXA1 and GATA3. Hence, the regulation of $F O X A 1$ and GATA3 probably occurs indirectly through the inhibition of ESR1 expression and/or other transcription factors. To obtain further evidence to support this hypothesis, we analyzed two known regulators of ESR1: miR-18a and miR-18b (Liu et al. 2009; additional file 2, Supplementary Tables 8 and 9 , see section on supplementary data given at the end of this article). The similarities between these three miRNAs were clear, and although the correlation and conservation were higher for miR-934 than for both miR-18a and miR-18b, the mirSVR score was slightly lower in the case of miR-934. Indeed, the energy value for miR-934 was between that of miR-18a and miR-18b. These results suggest that miR-934 may directly regulate ESR1 in a similar way to which it is regulated by miR-18a and miR-18b (additional file 2, Supplementary Table 9). Moreover, protein array data revealed a significant decrease in ESR1 phosphorylation $\left(P=7.29 \times 10^{-25}\right)$.

We also investigated the correlation between VGLL1 and all dataset genes. Interestingly, we found a positive association with luminal progenitor genes (GABRP, SLC6A14, FOXC1, PROM1, and BBOX1) located between the top ten genes more strongly correlated with VGLL1 expression. On the other hand, we found a strong negative correlation between VGLL1 expression and ER-associated genes (ESR1, C6ORF211, GATA3, and FOXA1; Table 3).

\section{VGLL1 protein expression in breast carcinomas}

We first analyzed the expression pattern of VGLL1 in positive controls (prostate carcinomas) and normal breast tissues. Nuclear staining was observed in neoplastic epithelial cells in some prostate cancer samples. Nuclear staining was also detected in lymphocytes. In addition, weak cytoplasmic staining was also found in some neoplastic epithelial cells. In normal breast tissue, we did not observe VGLL1 nuclear expression in either epithelial nor myoepithelial cells. In contrast, we found nuclear expression in some intraepithelial and stromal lymphocytes, detected by CD45 and/or CD3, and/or CD20 immunostaining (additional file 3, Supplementary Fig. 1, see section on supplementary data given at the end of this article). In addition, we analyzed a series of breast carcinoma cell lines representing luminal (MCF7 and
T47D), HER2 (BT474 and SKBR3), and basal subtypes (MCF10, MDA-MB-231, and BT459) by qRT-PCR, and found that VGLL1 mRNA expression was only detected in SKBR3 and BT474 cell lines (additional file 3, Supplementary Fig. 2). Our results were consistent with those reported by a previous study (Kao et al. 2009). These authors observed higher expression of VGLL1 in basal A breast cancer cell lines, and in some HER2-positive breast cancer cell lines, such as SKBR3, than in basal B breast carcinomas cell lines, such as MCF10, MDA-MB-231, and
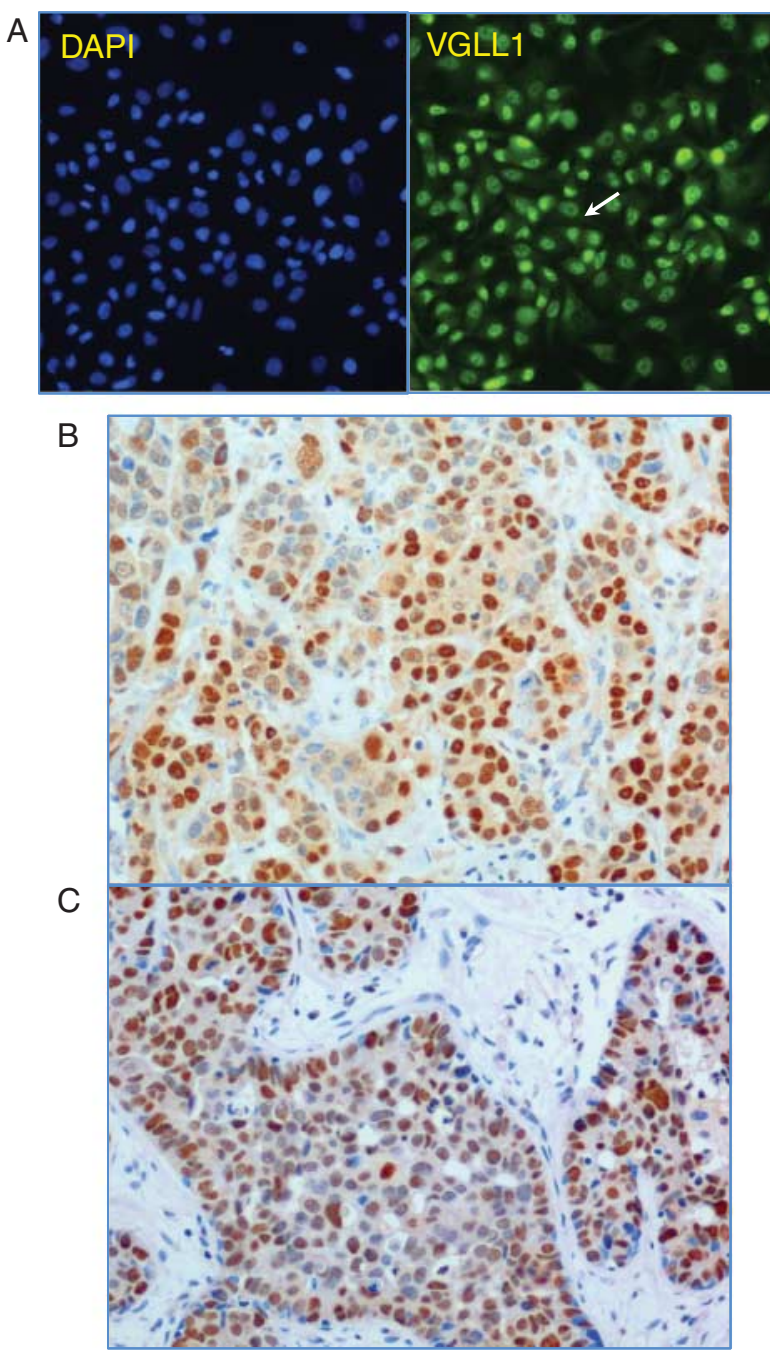

Figure 2

(A) Representative immunofluorescence staining of VGLL1 in the SKBR3 cell line as visualized by immunofluorescence microscopy. Nuclear expression is evident in most cells. In addition, weak cytoplasmic staining (arrow) is also seen in some cells. (B) Representative immunohistochemical nuclear staining of VGLL1 in a triple-negative invasive breast carcinoma (original magnification, $\times 200$ ). (C) Immunohistochemical staining of VGLL1 in a ductal carcinoma in situ (original magnification, $\times 200$ ).

Published by Bioscientifica Ltd. 
BT459. We carried out immunofluorescence analysis of VGLL1 in MCF-7 (negative control) and SKBR3 (positive control) to evaluate the pattern of protein expression and demonstrated intense nuclear VGLL1 expression and weak cytoplasmic expression in SKBR3 cells (Fig. 2A).

We examined the distribution of the VGLL1 protein in a total of 433 breast carcinomas by IHC carried out on TMA. Given that the only known function of VGLL1 reported to date is a transcriptional co-factor, we only evaluated its nuclear expression (Fig. $2 \mathrm{~B}$ and C). Tumors were considered positive if nuclear immunoreactivity was evident in $>5 \%$ of tumor cells.

VGLL1 expression was observed in 46 of 349 invasive breast carcinomas (13.18\%) and had no association with patient's age, tumor size, lymph node metastasis, or tumor stage (Table 1). However, significant associations were detected between VGLL1 expression and histological grade, ER, PR, and KI67 expression, as well as with the basal CK (CK5/6 and/or CK17) and c-Kit levels. We found no association between VGLL1 expression and HER2 and EGFR status. Thus, VGLL1 expression was more frequent among grade 3 invasive carcinomas that were ER- and PR-negative, that had a proliferation index $\geq 14 \%$ and basal CK expression. Based on these observations, we investigated whether VGLL1 nuclear expression was associated with different molecular phenotypes, as defined in the St Gallen consensus (Goldhirsch et al. 2011). VGLL1 was only occasionally expressed in luminal A $(0.70 \%)$ and B (5.60\%) tumors, but it was expressed in over $40 \%$ of TN breast carcinomas $(27 / 67)$. Tumors that overexpressed HER2 exhibited similar frequencies of VGLL1 expression (17\%) regardless of their ER expression. We then investigated whether there was a differential VGLL1 expression between $\mathrm{TN}$ breast carcinomas according to their expression of basal markers. Although the frequency of VGLL1 expression appeared to be higher in TN tumors with basal CKs and/or EGFR expression (45.70 vs $34.40 \%$ ), this difference was not statistically significant.

To confirm these results, we analyzed two additional series, one composed of $70 \mathrm{TN}$ sporadic breast carcinomas (additional file 2, Supplementary Table 2) and another of 14 BRCA1-associated carcinomas (including nine TN carcinomas) (additional file 2, Supplementary Table 3). We found that VGLL1 was expressed in $28.5 \%$ of sporadic and $55.5 \%$ of BRCA1-associated TN carcinomas. Considering the three series of invasive breast carcinomas examined in this study, we analyzed a total of $155 \mathrm{TN}$ breast carcinomas, 52 (33.5\%) of which expressed VGLL1.

Metaplastic carcinoma is one specific histological type of TN breast carcinomas that expresses basal markers and that is characterized by the acquisition of an epithelial-to-mesenchymal phenotype (Sarrio et al. 2008, Castilla et al. 2012). Of the TN breast carcinomas analyzed in this study, 16 were metaplastic carcinomas and they all expressed basal CKs and/or EGFR, while 6 (37.5\%) expressed VGLL1.

To determine whether VGLL1 was also expressed in the in situ component of invasive $\mathrm{TN}$ breast carcinomas (Fig. 2C), we analyzed the VGLL1 expression in wholetissue sections from ten samples (five VGLL1-positive and five VGLL1-negative TN breast carcinomas), in which both in situ and invasive components were adjacent in single sections. In all cases, we observed the same profile of VGLL1 expression in both carcinoma components by IHC.

\section{VGLL1 expression and prognosis}

A Kaplan-Meier survival analysis of 212 infiltrating breast carcinoma revealed a correlation between VGLL1-positive expression and reduced OS. The follow-up period ranged from 1 to 77 months, and the mean OS was 70.33 months for VGLL1-negative patients and 37.04 months for VGLL1positive patients $(P=0.0148$; additional file 3 , Supplementary Fig. 3 , see section on supplementary data given at the end of this article). In univariate Cox proportional hazard, analysis of OS VGLL1 expression was associated with adverse prognosis (Supplementary Table 10). Owing to the limited number of events, we selected the most significant variables for multivariate analysis. The association found between VGLL1 expression and poor prognosis is lost when adjusted for major prognostic factors. There were no significant differences in our cohort of patients on DFS rates between positive VGLL1 expression and negative VGLL1 expression $(P=0.067$; additional file 2, Supplementary Table 10).

\section{Discussion}

In this study, we have specifically evaluated the expression of miR-934 and VGLL1 in breast cancer and for the first time, we demonstrate an association between their expression and a TN-BL phenotype. When we examined the differences in miRNA expression between different groups of highly aggressive breast carcinomas, we identified 28 miRNAs that were differentially expressed between grade $3 \mathrm{ER}+/$ HER 2 - (comparable to luminal B carcinomas) and grade 3 TN IDCs. These findings were in line with the differential miRNA expression between luminal A and basal-like breast carcinomas found previously (Enerly et al. 2011), where 26 differentially expressed miRNAs

Published by Bioscientifica Ltd. 
almost perfectly separated the 2 subgroups: 13 upregulated and 13 downregulated in basal-like carcinomas. Among these 26 miRNAs, there were 11 miRNAs (miR-934, miR-9-3p/-5p, miR-135b, miR18a/b, miR-190, miR-149, miR-29c/29c*, and miR-342-3p) that were also differentially expressed in this study, and that may constitute the core miRNA signature of TN breast carcinomas.

The differential expression observed in our study demonstrates that TN breast carcinomas are characterized by the expression of miRNAs that downregulate ESR1 expression, such as let-7b (Zhao et al. 2011a,b, Quesne et al. 2012) and miR-18a/b (Leivonen et al. 2009, Liu et al. 2009, Yoshimoto et al. 2011). According to this observation, we found that miR-934 was the most significantly modulated miRNA in TN breast cancer, and confirmed this by our analyses of data from the TCGA database (2012). Interestingly, ESR1 and C6ORF211 were among the top ten genes most negatively correlated with miR-934 in the TCGA dataset. C6ORF211 is an open reading frame located immediately upstream of ESR 1 and its expression is strongly correlated with ESR1 in several publicly available datasets, suggesting transcriptional co-activation (Dunbier et al. 2011). Although the function of miR-934 has not been determined, it is likely to be involved in direct ESR1 modulation. Our in silico comparison between miR-934 and two proven ESR1 regulators, miR-18a and miR-18b, revealed significant similarities in terms of gene correlations, seed sequence conservation among species, mirSVR scores and energy values. Moreover, among 59 miRNAs that were identified that may target the ESR1 $3^{\prime}$-UTR (using a luciferase reporter plasmid carrying the entire 3'-UTR of ESR1), miR-934 most strongly suppressed the luciferase signal (Xiong et al. 2010).

The significant modulation of miR-934 expression in TN breast carcinomas drew our attention to VGLL1 gene, as miR-934 is located in intron 4 of VGLL1. At present, the role of $V G L L 1$ in cancer is unknown, although the oncogenic potential of VGLL1 has been demonstrated in one study reported by Pobbati et al. (2012). As well as a structural and functional similarity between the VGLL1TEAD complex and that formed by TEAD and YAP, it was also demonstrated that VGLL1-TEAD complexes promote anchorage-independent cell proliferation. In this study, we first demonstrated that VGLL1 mRNA was overexpressed in TN breast carcinomas as opposed to ER+ grade 3 IDCs, and that its expression was correlated with that of miR-934, suggesting at least a partial co-regulation.

Through IHC, we demonstrated for the first time that nuclear expression of VGLL1 protein is associated with TN and basal-like phenotypes. A detailed analysis of several publicly available datasets revealed that VGLL1 expression is consistently associated with grade 3 breast carcinomas with a basal-like phenotype (van 't Veer et al. 2002, Farmer et al. 2005, Loi et al. 2007, Lehmann et al. 2011, Moreno-Bueno et al. 2011, Sabatier et al. 2011), confirming our results. Moreover, in the TCGA dataset, VGLL1 was not only significantly upregulated in basal-like carcinomas with respect to other molecular subtypes but also, it was the gene most strongly correlated with miR-934, supporting the hypothesis of transcriptional coregulation. Although the mechanisms of miR-934/VGLL1 overexpression are at present unknown, the analysis of TCGA data excluded copy number variation as an important cause.

$\mathrm{TN}$ and basal-like breast carcinomas are morphologically and molecularly heterogeneous. A transcriptional analysis of $587 \mathrm{TN}$ carcinomas reported VGLL1 overexpression in the basal-like 1 carcinoma subtype (Lehmann et al. 2011). This subtype exhibited enhanced expression of cell cycle and DNA damage response genes, and representative cell lines of this group, identified as basal A, also expressed VGLL1 at higher levels (Kao et al. 2009). Interestingly, these cell lines exhibited an enhanced response to cisplatin and poly ADP ribose polymerase (PARP) inhibitors when compared with cell lines belonging to other TN subtypes.

Despite being a small sample size, our results suggest that VGLL1 upregulation is more frequent in TN BRCA1associated breast carcinomas. Indeed, VGLL1 was one of the most strongly upregulated genes in ER-negative BRCA1-positive as opposed to ER-negative sporadic carcinomas (van't Veer et al. 2002). Moreover, cell lines derived from carriers of BRCA1 mutations are of the basal A/basal 1 subtype (Kao et al. 2009, Lehmann et al. 2011), characterized by VGLL1 overexpression. VGLL1 also appears to be a marker of luminal progenitor cells, the cells originating BRCA1-associated carcinomas, and a large number of sporadic basal-like carcinomas. Thus, VGLL1 was one of the genes in a luminal progenitor signature (Lim et al. 2009), along with 5 (GABRP, SLC6A14, FOXC1, PROM1, and BBOX1) of the ten genes that were most strongly correlated with VGLL1 in the TCGA dataset. Interestingly, GABRP, which was previously associated with basal-like breast carcinomas, was the most strongly correlated with VGLL1 in the TCGA dataset and it was one of the most strongly upregulated genes in the aforementioned luminal progenitor signature (Lim et al. 2009). Moreover, PROM1 (CD133), which is also correlated with VGLL1 in different datasets (Bertheau et al. 2007, Kao et al. 2009), is expressed in a subpopulation of cells

Published by Bioscientifica Ltd 
with cancer stem cell properties in BRCA1-deficient mouse mammary tumors (Wright et al. 2008).

A role of EZH2 and GATA3 in the maintenance of a luminal progenitor phenotype has recently been reported (Granit et al. 2012). Indeed, EZH2 was shown to promote a bi-lineage identity in basal-like breast cancer cells (Granit et al. 2012), and luminal progenitor-associated genes, including VGLL1, were preferentially downregulated in EZH2-silenced cells and upregulated in EZH2overexpressing cells. By contrast, GATA3, a driver of luminal differentiation, performs an opposite function to that of EZH2, suppressing bi-lineage identity and luminal-progenitor genes expression. Indeed, GATA3 levels increase upon EZH2 silencing, mediating a decrease in bi-lineage cell numbers (Granit et al. 2012). Interestingly, the three genes with the greatest negative correlation with VGLL1 and miR-934 in the TCGA dataset were GATA3, ESR1, and FOXA1, which form a functional enhanceosome that regulates the genes driving core ER $\alpha$ functions and that co-operatively modulates the transcriptional networks previously ascribed to ER $\alpha$ alone (Kong et al. 2011). Gain and loss of function in vitro studies are currently in progress in our group, using miR-934/VGLL1negative (MCF7 and T47d) and VGLL1-positive (SKBR3 and MDA-MB-468) cell lines, respectively, that will confirm the suggested role of miR-934 in the modulation of ESR1 expression and the maintenance of a luminal progenitor phenotype.

In conclusions, we demonstrate for the first time that $V G L L 1$, a transcriptional coactivator with structural similarities to TAZ and YAP, is mainly expressed in a subset of sporadic and BRCA1-associated TN basal-like breast carcinomas. An analysis of several publicly available datasets suggests that VGLL1 is preferentially expressed in tumors and breast cancer cell lines with luminal progenitor features, these exhibiting an enhanced response to cisplatin and PARP inhibitors. Moreover, VGLL1 expression was correlated with the expression of its intronic miRNA, miR-934, a potential direct modulator of ESR1 expression. Together, our findings indicate that the coordinated expression of VGLL1 and miR-934 may be involved in the maintenance of the luminal progenitor phenotype, at least in part mediated by their direct modulation of ESR1. These finding might be useful to select specific therapies among TN breast carcinomas.

Future studies should analyze whether or not tumors expressing VGLL1 might represent a subset of luminal progenitor-enriched breast carcinomas more sensible to specific treatment, such as PARP1 inhibitors and cisplatin.

\section{Supplementary data}

This is linked to the online version of the paper at http://dx.doi.org/10.1530/ ERC-13-0485.

\section{Declaration of interest}

The authors declare that there is no conflict of interest that could be perceived as prejudicing the impartiality of the research reported.

\section{Funding}

This work was supported by grants from the Instituto de Salud Carlos III (ISCIII, grant numbers PI08/0971 and PI13/02477), co-financed by the European Development Regional Fund ('A way to achieve Europe': grant numbers RD06/0020/0013, RD09/0076/00085, and RD12/0036/0064) and by the Junta de Andalucía (Consejería de Salud, PI0581/2009). M Á Castilla is a $\mathrm{PhD}$ researcher funded by the ISCIII (RD06/0020/0013). M R Atienza is a MD researcher funded by the ISCIII (Rio Hortega program). J Daz-Martín and J M Rosa-Rosa are PhD researchers funded by the Consejería de Salud (PI0581/2009) and the ISCIII (RD12/0036/0064) respectively. L Romero-Pérez is a PhD student and a recipient of a PFIS fellowship (grant number F109/00193). M L Pecero works as a lab technician and is supported by the ISCIII (PI080971).

\section{Author contribution statement}

M Á Castilla and J Palacios conceived and designed the experiments. J Palacios, M Á López-García, M Á Castilla, M R Atienza, J Benítez, and J Díaz-Martín provided the tumor samples and clinical data. J Palacios, M Á Castilla, M Á López-García, and B Vieites performed the pathological characterization and immunohistochemical samples. M Á Castilla, J M RosaRosa, M L Pecero, L Romero-Pérez, and A Calcabrini carried out the experiments. M Á Castilla, J Palacios, J M Rosa-Rosa, and M Á López-García analyzed and interpreted the data. M Á Castilla and J Palacios wrote the paper with additional input from J M Rosa-Rosa. All authors reviewed the results and discussion.

\section{Acknowledgements}

The authors thank Juan Manuel Praena (Statistics, Methodology and Research Evaluation Unit, Andalusian Public Foundation for Health Research Management in Seville) and Javier Zamora (Clinical Biostatistics Unit, Hospital Ramón y Cajal-IRYCIS, Madrid) for help with the statistical analyses.

\section{References}

Badve S, Dabbs DJ, Schnitt SJ, Baehner FL, Decker T, Eusebi V, Fox SB, Ichihara S, Jacquemier J, Lakhani SR et al. 2011 Basal-like and triplenegative breast cancers: a critical review with an emphasis on the implications for pathologists and oncologists. Modern Pathology 24 157-167. (doi:10.1038/modpathol.2010.200)

Bertheau P, Turpin E, Rickman DS, Espie M, de Reynies A, Feugeas JP, Plassa LF, Soliman H, Varna M, de Roquancourt A et al. 2007 Exquisite sensitivity of TP53 mutant and basal breast cancers to a dose-dense epirubicin-cyclophosphamide regimen. PLoS Medicine 4 e90. (doi:10.1371/journal.pmed.0040090)

Castilla MA, Moreno-Bueno G, Romero-Perez L, Van De Vijver K, Biscuola M, Lopez-Garcia MA, Prat J, Matias-Guiu X, Cano A, Oliva E et al. 2011 Micro-RNA signature of the epithelial-mesenchymal transition in endometrial carcinosarcoma. Journal of Pathology $\mathbf{2 2 3}$ 72-80. (doi:10.1002/path.2802) 
Castilla MA, Diaz-Martin J, Sarrio D, Romero-Perez L, Lopez-Garcia MA, Vieites B, Biscuola M, Ramiro-Fuentes S, Isacke CM \& Palacios J 2012 MicroRNA-200 family modulation in distinct breast cancer phenotypes. PLoS ONE 7 e47709. (doi:10.1371/journal.pone.0047709)

Cerami E, Gao J, Dogrusoz U, Gross BE, Sumer SO, Aksoy BA, Jacobsen A Byrne CJ, Heuer ML, Larsson E et al. 2012 The cBio cancer genomics portal: an open platform for exploring multidimensional cancer genomics data. Cancer Discovery 2 401-404. (doi:10.1158/2159-8290. CD-12-0095)

Dunbier AK, Anderson H, Ghazoui Z, Lopez-Knowles E, Pancholi S, Ribas R, Drury S, Sidhu K, Leary A, Martin LA et al. 2011 ESR1 is co-expressed with closely adjacent uncharacterised genes spanning a breast cancer susceptibility locus at 6q25.1. PLoS Genetics 7 e1001382. (doi:10.1371/ journal.pgen.1001382)

Dweep H, Sticht C, Pandey P \& Gretz N 2011 miRWalk - database: prediction of possible miRNA binding sites by 'walking' the genes of three genomes. Journal of Biomedical Informatics 44 839-847. (doi:10.1016/j.jbi.2011.05.002)

Enerly E, Steinfeld I, Kleivi K, Leivonen SK, Aure MR, Russnes HG, Ronneberg JA, Johnsen H, Navon R, Rodland E et al. 2011 miRNAmRNA integrated analysis reveals roles for miRNAs in primary breast tumors. PLOS ONE 6 e16915. (doi:10.1371/journal.pone.0016915)

Farmer P, Bonnefoi H, Becette V, Tubiana-Hulin M, Fumoleau P, Larsimont D, Macgrogan G, Bergh J, Cameron D, Goldstein D et al. 2005 Identification of molecular apocrine breast tumours by microarray analysis. Oncogene $\mathbf{2 4}$ 4660-4671. (doi:10.1038/sj.onc.1208561)

Goldhirsch A, Wood WC, Coates AS, Gelber RD, Thurlimann B \& Senn HJ 2011 Strategies for subtypes - dealing with the diversity of breast cancer: highlights of the St. Gallen International Expert Consensus on the Primary Therapy of Early Breast Cancer 2011. Annals of Oncology 22 1736-1747. (doi:10.1093/annonc/mdr304)

Granit RZ, Gabai Y, Hadar T, Karamansha Y, Liberman L, Waldhorn I, Gat-Viks I, Regev A, Maly B, Darash-Yahana M etal. 2012 EZH2 promotes a bi-lineage identity in basal-like breast cancer cells. Oncogene 32 3886-3895. (doi:10.1038/onc.2012.390)

Kao J, Salari K, Bocanegra M, Choi YL, Girard L, Gandhi J, Kwei KA, Hernandez-Boussard T, Wang P, Gazdar AF et al. 2009 Molecular profiling of breast cancer cell lines defines relevant tumor models and provides a resource for cancer gene discovery. PLOS ONE 4 e6146. (doi:10.1371/journal.pone.0006146)

Kong SL, Li G, Loh SL, Sung WK \& Liu ET 2011 Cellular reprogramming by the conjoint action of $\mathrm{ER} \alpha$, FOXA1, and GATA3 to a ligand-inducible growth state. Molecular Systems Biology 7 526. (doi:10.1038/msb. 2011.59)

Lamar JM, Stern P, Liu H, Schindler JW, Jiang ZG \& Hynes RO 2012 The Hippo pathway target, YAP, promotes metastasis through its TEAD-interaction domain. PNAS 109 E2441-E2450. (doi:10.1073/ pnas.1212021109)

Lehmann BD, Bauer JA, Chen X, Sanders ME, Chakravarthy AB, Shyr Y \& Pietenpol JA 2011 Identification of human triple-negative breast cancer subtypes and preclinical models for selection of targeted therapies. Journal of Clinical Investigation 121 2750-2767. (doi:10.1172/JCI45014)

Leivonen SK, Makela R, Ostling P, Kohonen P, Haapa-Paananen S, Kleivi K, Enerly E, Aakula A, Hellstrom K, Sahlberg N et al. 2009 Protein lysate microarray analysis to identify microRNAs regulating estrogen receptor signaling in breast cancer cell lines. Oncogene 28 3926-3936. (doi:10.1038/onc.2009.241)

Lim E, Vaillant F, Wu D, Forrest NC, Pal B, Hart AH, Asselin-Labat ML, Gyorki DE, Ward T, Partanen A et al. 2009 Aberrant luminal progenitors as the candidate target population for basal tumor development in BRCA1 mutation carriers. Nature Medicine 15 907-913. (doi:10.1038/ $\mathrm{nm} .2000)$

Liu WH, Yeh SH, Lu CC, Yu SL, Chen HY, Lin CY, Chen DS \& Chen PJ 2009 MicroRNA-18a prevents estrogen receptor- $\alpha$ expression, promoting proliferation of hepatocellular carcinoma cells. Gastroenterology 136 683-693. (doi:10.1053/j.gastro.2REF16=10.1038/onc.2009.241)

http://erc.endocrinology-journals.org DOI: 10.1530/ERC-13-0485
(C) 2014 Society for Endocrinology Printed in Great Britain
Loi S, Haibe-Kains B, Desmedt C, Lallemand F, Tutt AM, Gillet C, Ellis P, Harris A, Bergh J, Foekens JA et al. 2007 Definition of clinically distinct molecular subtypes in estrogen receptor-positive breast carcinomas through genomic grade. Journal of Clinical Oncology 25 1239-1246. (doi:10.1200/JCO.2006.07.1522)

Moreno-Bueno G, Salvador F, Martin A, Floristan A, Cuevas EP, Santos V, Montes A, Morales S, Castilla MA, Rojo-Sebastian A et al. 2011 Lysyl oxidase-like 2 (LOXL2), a new regulator of cell polarity required for metastatic dissemination of basal-like breast carcinomas. EMBO Molecular Medicine 3 528-544. (doi:10.1002/emmm.201100156)

Palacios J, Honrado E, Osorio A, Cazorla A, Sarrio D, Barroso A, Rodriguez S, Cigudosa JC, Diez O, Alonso C et al. 2005 Phenotypic characterization of BRCA1 and BRCA2 tumors based in a tissue microarray study with 37 immunohistochemical markers. Breast Cancer Research and Treatment 90 5-14. (doi:10.1007/s10549-004-1536-REF21=10.1007/s10549-0041536-0)

Perou CM, Sorlie T, Eisen MB, van de Rijn M, Jeffrey SS, Rees CA, Pollack JR, Ross DT, Johnsen H, Akslen LA et al. 2000 Molecular portraits of human breast tumours. Nature 406 747-752. (doi:10.1038/35021093)

Pobbati AV, Chan SW, Lee I, Song H \& Hong W 2012 Structural and functional similarity between the Vgll1-TEAD and the YAP-TEAD complexes. Structure 20 1135-1140. (doi:10.1016/j.str.2012.04.004)

Quesne JL, Jones J, Warren J, Dawson SJ, Ali HR, Bardwell H, Blows F, Pharoah P \& Caldas C 2012 Biological and prognostic associations of miR-205 and let-7b in breast cancer revealed by in situ hybridization analysis of micro-RNA expression in arrays of archival tumour tissue. Journal of Pathology 227 306-314. (doi:10.1002/path.3983)

Sabatier R, Finetti P, Cervera N, Lambaudie E, Esterni B, Mamessier E, Tallet A, Chabannon C, Extra JM, Jacquemier J et al. 2011 A gene expression signature identifies two prognostic subgroups of basal breast cancer. Breast Cancer Research and Treatment 126 407-420. (doi:10.1007/s10549010-0897-9)

Sarrio D, Rodriguez-Pinilla SM, Hardisson D, Cano A, Moreno-Bueno G \& Palacios J 2008 Epithelial-mesenchymal transition in breast cancer relates to the basal-like phenotype. Cancer Research 68 989-997. (doi:10.1158/0008-5472.CAN-07-2017)

Sorlie T, Perou CM, Tibshirani R, Aas T, Geisler S, Johnsen H, Hastie T, Eisen MB, van de Rijn M, Jeffrey SS et al. 2001 Gene expression patterns of breast carcinomas distinguish tumor subclasses with clinical implications. PNAS 98 10869-10874. (doi:10.1073/pnas.191367098)

Sorlie T, Tibshirani R, Parker J, Hastie T, Marron JS, Nobel A, Deng S, Johnsen H, Pesich R, Geisler S et al. 2003 Repeated observation of breast tumor subtypes in independent gene expression data sets. PNAS 100 8418-8423. (doi:10.1073/pnas.0932692100)

The Cancer Genome Atlas Network 2012 Comprehensive molecular portraits of human breast tumours. Nature 490 61-70. (doi:10.1038/ nature11412)

Toft DJ \& Cryns VL 2011 Minireview: Basal-like breast cancer: from molecular profiles to targeted therapies. Molecular Endocrinology 25 199-211. (doi:10.1210/me.2010-0164)

Vaudin P, Delanoue R, Davidson I, Silber J \& Zider A 1999 TONDU (TDU), a novel human protein related to the product of vestigial (vg) gene of Drosophila melanogaster interacts with vertebrate TEF factors and substitutes for Vg function in wing formation. Development 126 4807-4816.

van 't Veer LJ, Dai H, van de Vijver MJ, He YD, Hart AA, Mao M, Peterse HL, van der Kooy K, Marton MJ, Witteveen AT et al. 2002 Gene expression profiling predicts clinical outcome of breast cancer. Nature $\mathbf{4 1 5}$ 530-536. (doi:10.1038/415530a)

Wright MH, Calcagno AM, Salcido CD, Carlson MD, Ambudkar SV \& Varticovski L 2008 Brca1 breast tumors contain distinct CD44+/ CD24 - and CD133 + cells with cancer stem cell characteristics. Breast Cancer Research 10 R10. (doi:10.1186/bcr1855)

Xiong J, Yu D, Wei N, Fu H, Cai T, Huang Y, Wu C, Zheng X, Du Q, Lin D et al. 2010 An estrogen receptor $\alpha$ suppressor, microRNA-22, is downregulated in estrogen receptor $\alpha$-positive human breast cancer cell 
lines and clinical samples. FEBS Journal 277 1684-1694. (doi:10.1111/ j.1742-4658.2010.07594.x)

Yoshimoto N, Toyama T, Takahashi S, Sugiura H, Endo Y, Iwasa M, Fujii Y \& Yamashita H 2011 Distinct expressions of microRNAs that directly target estrogen receptor $\alpha$ in human breast cancer. Breast Cancer Research and Treatment 130 331-339. (doi:10.1007/s10549-011-1672-2)

Zecca M \& Struhl G 2010 A feed-forward circuit linking wingless, fatdachsous signaling, and the Warts-Hippo pathway to Drosophila wing growth. PLoS Biology 8 e1000386. (doi:10.1371/journal.pbio.1000386)
Zhao Y, Deng C, Lu W, Xiao J, Ma D, Guo M, Recker RR, Gatalica Z, Wang Z \& Xiao GG 2011a let-7 microRNAs induce tamoxifen sensitivity by downregulation of estrogen receptor $\alpha$ signaling in breast cancer. Molecular Medicine 17 1233-1241. (doi:10.2119/ molmed.2010.00225)

Zhao Y, Deng C, Wang J, Xiao J, Gatalica Z, Recker RR \& Xiao GG $2011 b$ Let-7 family miRNAs regulate estrogen receptor $\alpha$ signaling in estrogen receptor positive breast cancer. Breast Cancer Research and Treatment 127 69-80. (doi:10.1007/s10549-010-0972-2)

Received in final form 27 May 2014

Accepted 29 May 2014

Made available online as an Accepted Preprint

2 June 2014
Published by Bioscientifica Ltd. 